\title{
Tuning the properties of carbon fiber-reinforced poly(phenylene sulphide) laminates via incorporation of inorganic nanoparticles
}

\author{
Ana M. Díez-Pascual, Mohammed Naffakh
}

\begin{abstract}
A B S T R A C T
Novel carbon fiber (CF)-reinforced poly(phenylene sulphide) (PPS) laminates incorporating inorganic fullerene-like tungsten disulfide (IF-WS ${ }_{2}$ ) nanoparticles were prepared via melt-blending and hot-press processing. The influence of the IF-WS $\mathrm{WS}_{2}$ on the morphology, thermal, mechanical and tribological properties of PPS/CF composites was investigated. Efficient nanoparticle dispersion within the matrix was attained without using surfactants. A progressive rise in thermal stability was found with increasing IF-WS $_{2}$ loading, as revealed by thermogravimetric analysis. The addition of low nanoparticle contents retarded the crystallization of the matrix, whereas concentrations equal or higher than $1.0 \mathrm{wt} \%$ increased both the crystallization temperature and degree of crystallinity compared to those of PPS/CF. Mechanical tests indicated that with only $1.0 \mathrm{wt} \%$ IF-WS 2 the flexural modulus and strength of PPS/CF improved by 17 and $14 \%$, respectively, without loss in toughness, ascribed to a synergistic effect between the two fillers. A significant enhancement in the storage modulus and glass transition temperature was also observed. Moreover, the wear rate and coefficient of friction strongly decreased, attributed to the lubricant role of the IF-WS 2 combined with their reinforcing effect. These inorganic nanoparticles show great potential to improve the mechanical and tribological properties of conventional thermoplastic/CF composites for structural applications.
\end{abstract}

\section{Introduction}

Carbon fiber-reinforced plastics (CFRP) have been manufactured during the last decades to develop materials which combine high stiffness and strength with low density. This combination of properties has led to a strong increase in the use of these composites for applications in the aeronautic [1], automotive [2] and energy sectors [3], replacing traditional materials such as steel, aluminum and wood, amongst others. Thermoplastics (TPs) offer some advantages over thermosetting resins, namely improved chemical and impact resistance as well as a wider application temperature range. Moreover, they have a very low level of moisture absorption, hence their mechanical properties are retained under harsh environmental conditions. Another key aspect is shelf life; unlike thermosets, TPs can be easily stored for long periods of time without loss of performance or ability to process. TP materials with complex shapes can be easily manufactured in a few steps through a thermoforming process, and offer the possibility to be remelted, providing recycling opportunities. They can be joined successfully in a number of different ways, including mechanical fastenings, ultrasonic assembly, metal inserts, snap fits, heat welding and sealing, adhesive bonding, etc [4]. Although plastics are initially tougher than thermosets, once damaged they are more difficult to repair. Other disadvantages of TPs are their higher price and the need of high processing pressures and expensive equipment, as well as significant energy cost to heat the tooling.

The most widely used high-performance TPs are poly(ether ether ketone) (PEEK), poly(phenylene sulphide) (PPS) and poly(etherimide) (PEI). PPS is a semicrystalline polymer composed of phenyl rings and sulfur atoms that possesses outstanding mechanical and thermal properties. Composite structures made of PPS remain hard, impact-resistant, stiff and dimensionally stable even when exposed to temperature variations of more than $100^{\circ} \mathrm{C}$. They are also resistant to aggressive media, possess inherent flame retardancy, minimum water absorption, anti-aging and excellent friction properties [5]. The most important application areas are the automobile, aeronautic and electronics industries [6]. Carbon fiber- 
reinforced PPS (PPS/CF) is mainly used in aircraft structural applications, such as in J-Nose wing substructures of the Airbus A340$500 / 600$ [7]. To the best of our knowledge, very few studies deal with the behavior of carbon fabrics reinforced PPS composites $[8,9]$.

Conventional CF-reinforced composites usually exhibit poor fracture toughness and delamination resistance. To address these issues, additional nanoscale fillers such as carbon nanotubes (CNTs) [10] or inorganic nanoparticles [11] have been mixed with CFs to reinforce polymer matrices. Their extremely high specific surface area facilitates the generation of large amount of interphase in the composite and strong interactions between the fillers and the matrix. In particular, the addition of nanoclays to CFRPs has been reported to improve the damping properties, fatigue life, toughness and wear resistance [12-14]. The synergistic effect of CFs with the inorganic particles is supposed to be the main reason for the observed mechanical enhancement. A new strategy is the incorporation of nanosized inorganic fullerene-like tungsten disulfide $\left(\mathrm{IF}^{\left.-\mathrm{WS}_{2}\right)}\right.$ nanoparticles [15], due to their extraordinary properties, such as high modulus and low friction coefficient, attributed to their small size, closed structure, and chemical inertness that make them suitable candidates for composite reinforcement. They exhibit excellent solid lubricant behavior [15], decreasing the viscosity of TPs and improving their tribological properties. Moreover, they are much cheaper than CNTs and can be efficiently dispersed within polymer matrices up to concentrations of $8 \mathrm{wt} \%$ [16].

In a previous study [16] IF-WS ${ }_{2}$ nanoparticles were used to prepare PPS/IF-WS 2 nanocomposites, and the results demonstrated significant improvements in the mechanical properties of the matrix at low loadings. The main aim of the current work is to investigate the effect of these nanofillers on the properties of conventional PPS/CF laminates. The multiscale composites were prepared through simple melt-blending and hot-press processing, techniques that are environmentally friendly and easy to scale up, which would be interesting for potential industrial applications. These hybrid composites are composed of alternating layers of PPS/ IF-WS 2 films and carbon fiber fabrics, combining the properties of all the constituents; hence, they are expected to have improved performance in comparison with binary composites. The influence of the IF-WS 2 concentration on the degree of crystallinity, thermal, mechanical and tribological properties of these multifunctional PPS based laminates is discussed.

\section{Experimental section}

\subsection{Materials}

PPS (Fortron 02054P4) was kindly supplied by Ticona in pellet form. The polymer was dried at $100{ }^{\circ} \mathrm{C}$ for $14 \mathrm{~h}$ and stored in a dry environment before blending. Inorganic fullerene-like tungsten disulfide $\left(\mathrm{IF}-\mathrm{WS}_{2}\right.$ ) nanoparticles (NanoLub ${ }^{\mathrm{TM}}$ ) were provided by Nanomaterials (Israel). They exhibit a quasi-spherical shape with an average aspect ratio of 1.4. Standard modulus carbon fiber fabrics 5-harness satin (fabric reference G0926), with an areal weight of $370 \pm 14 \mathrm{~g} / \mathrm{m}^{2}$, was provided by Hexcell Reinforcements (France). Some physical and mechanical properties of the materials used for the preparation of the laminates are collected in Table 1.

\subsection{Preparation of PPS/IF-WS $S_{2}$ films}

Firstly, different concentrations of IF-WS $2(0.1,0.5 .1 .0$ and $2.0 \mathrm{wt}$ $\%$ ) were incorporated into PPS via melt-blending using a Haake Rheocord 90 extruder operating at $320 \pm 5^{\circ} \mathrm{C}$, with a rotor speed of $150 \mathrm{rpm}$ and mixing times of $20 \mathrm{~min}$. Then, the PPS/IF-WS extrudate was used to fabricate films with a thickness of $\sim 0.2 \mathrm{~mm}$. The films were made in a hot-press at the same temperature under high pressure. A brass frame was employed to control the dimensions, and two flat brass plates were used as top and bottom surfaces to guarantee uniform thickness of the thin films.

\subsection{Preparation of PPS/IF-WS $/$ CF laminates}

The composite laminates were fabricated by alternatively placing 4 plies of carbon fiber fabric between $5 \mathrm{PPS} / \mathrm{IF}^{-\mathrm{WS}_{2}}$ films using hot-compression, and were consolidated at $320 \pm 5^{\circ} \mathrm{C}$ under successive pressure dwell steps of 5,40 and 130 bars, for periods of 30,25 and $5 \mathrm{~min}$, respectively. The pressure steps were optimized to improve fiber impregnation and minimize internal porosity. The heating rate to the dwell temperature was $\sim 5{ }^{\circ} \mathrm{C} / \mathrm{min}$, and the cooling to room temperature was undertaken at a rate $\leq 3{ }^{\circ} \mathrm{C} / \mathrm{min}$. The resulting laminates had a nominal thickness of $1 \pm 0.1 \mathrm{~mm}$, a resin content of $35 \pm 2 \mathrm{wt} \%$, a fiber volume fraction of $0.59 \pm 0.01$ an average density of $1.62 \pm 0.03 \mathrm{~g} / \mathrm{cm}^{3}$ and a void content $<2 \%$, which was determined from the experimental density and that calculated from the weight fraction of the components and their individual densities. For the different characterizations the laminates were cut into smaller pieces with a diamond cutting wheel.

\subsection{Materials characterization}

The morphology of the fracture and worn surfaces of the laminates was investigated with a Philips XL30 scanning electron microscope (SEM) operating at $25 \mathrm{kV}$ using a lanthanum hexaboride $\left(\mathrm{LaB}_{6}\right)$ filament. Samples were mounted on an aluminum stub and coated with a $\sim 5 \mathrm{~nm} \mathrm{Au/Pd}$ overlayer in a Balzers SDC evaporator to avoid charge accumulation during electron irradiation.

DSC experiments were performed on a Mettler TA4000 differential scanning calorimeter, equipped with a DSC-30 oven with automatic temperature control, operating under nitrogen flow. Samples of $\sim 20 \mathrm{mg}$ sealed in aluminum pans were heated to $320^{\circ} \mathrm{C}$ for $5 \mathrm{~min}$, cooled to ambient temperature and then reheated to $320^{\circ} \mathrm{C}$, all steps at the same rate of $10^{\circ} \mathrm{C} / \mathrm{min}$. Glass transition temperatures were determined as the mid-point of the baseline shift, and the melting and crystallization temperatures were taken as the peak maxima and minima in the calorimetric curves, respectively. The degree of crystallinity $\chi_{c, m}(\%)$ was estimated according to the equation: $\chi_{c, m}=\Delta H_{c, m, \mathrm{PPS}} /\left(\Delta H_{m \text {,PPS }}^{\circ} \times w_{\mathrm{PPS}}\right)$, where $\Delta H_{m, \text { PPS }}^{\circ}$ is the heat of fusion of a $100 \%$ crystalline PPS $(\sim 80 \mathrm{~J} / \mathrm{g})$ [5] $\Delta H_{c, m, P P S}$ is the apparent crystallization or melting enthalpy of PPS and $w_{\text {PPS }}$ is the weight fraction of the polymer.

The thermal stability of the laminates and their residual weight were analyzed by thermogravimetric analysis (TGA) using a Mettler TA-4000/TG-50 thermobalance at a heating rate of $10^{\circ} \mathrm{C} / \mathrm{min}$. The

Table 1

Properties of the raw materials used for the preparation of the laminates $[17,18]$.

\begin{tabular}{|c|c|c|c|c|c|c|}
\hline Material & $d_{25}{ }^{\circ} \mathrm{C}\left(\mathrm{g} / \mathrm{cm}^{3}\right)$ & $D(\mu \mathrm{m})$ & $T_{\mathrm{g}}\left({ }^{\circ} \mathrm{C}\right)$ & $T_{m}\left({ }^{\circ} \mathrm{C}\right)$ & $E_{25}{ }^{\circ} \mathrm{C}(\mathrm{GPa})$ & $\sigma_{y}(\mathrm{MPa})$ \\
\hline PPS & 1.35 & - & 90 & 285 & 2.2 & 63 \\
\hline CF & 1.77 & 7 & - & - & 234 & 3650 \\
\hline IF-WS ${ }_{2}$ & 7.50 & 0.08 & - & - & 150 & 2420 \\
\hline
\end{tabular}

$D$ : average diameter; $T_{m}$ : melting temperature; $E$ : Young's modulus; $\sigma_{y}$ : tensile strength. 
temperature was scanned from room temperature to $800^{\circ} \mathrm{C}$ under both nitrogen and dry air atmospheres. Experiments were carried out on samples with an average mass of $150 \mathrm{mg}$, and the purge air flow rate was $50 \mathrm{ml} / \mathrm{min}$.

A dynamic mechanical analyzer (Mettler DMA 861) was used to measure the storage modulus, loss modulus and tan $\delta$ of the materials by heating from -100 to $250{ }^{\circ} \mathrm{C}$ with a ramping rate of $2{ }^{\circ} \mathrm{C} / \mathrm{min}$, at frequencies of $0.1,1$ and $10 \mathrm{~Hz}$. A dynamic force of $6 \mathrm{~N}$ was used oscillating at fixed frequency and amplitude of $30 \mu \mathrm{m}$.

Tensile and flexural properties were measured with an INSTRON 4204 mechanical tester at room temperature and $50 \pm 5 \%$ relative humidity (RH), using a crosshead speed of $1 \mathrm{~mm} / \mathrm{min}$ and a load cell of $1 \mathrm{kN}$. Tensile specimens (Type $\mathrm{V}$ ) and rectangular flexural coupons were employed, according to UNE-EN ISO 527-1 and 178 standards, respectively. All the samples were conditioned for $24 \mathrm{~h}$ before the measurements. The data reported correspond to the average of the results for 5 specimens.

Charpy notched impact strength measurements were carried out using a CEAST Fractovis dart impact tester. A hammer mass of $1.096 \mathrm{~kg}$ impacted at a constant velocity of $3.60 \mathrm{~m} \mathrm{~s}^{-1}$ (giving a total kinetic energy at impact of $7.10 \mathrm{~J}$ ) on notched specimen bars with a $V$-shape notch tip radius $=0.25 \mathrm{~mm}$, as described in the UNE-EN ISO 179 standard. Measurements were performed at $23 \pm 2{ }^{\circ} \mathrm{C}$ and $50 \pm 5 \%$ RH. At least 6 specimens were tested for each type of composite to check for repeatability.

A micro-Vickers hardness tester (Innovatest ${ }^{(} 422 \mathrm{~A}$ ) was used to evaluate the microhardness of the laminates. The specimens were subjected to a load of $50 \mathrm{~g}$ during $15 \mathrm{~s}$ under controlled ambient conditions. The diagonals of each indentation were measured 3 times to obtain average values.

Pin-on-disk tests were performed on a Microtest MT 400-98 apparatus, using a $6 \mathrm{~mm}$ diameter $100 \mathrm{Cr} 6$ steel ball like pattern slide. Measurements were carried out under a constant load of $5 \mathrm{~N}$ at a rotation speed of $375 \mathrm{rpm}$. The equipment was placed in an isolated box to control the atmospheric conditions, and the experiments were performed in air at $24+2{ }^{\circ} \mathrm{C}$ and $22+2 \% \mathrm{RH}$. The wear experienced by the flat substrate was determined through the measurement of the wear-track profile by using a profilometer with a resolution of $\sim 10 \mathrm{~nm}$. Each tribological test was repeated 3 times to minimize data scattering and the average value is reported.

\section{Results and discussion}

\subsection{Morphological observations}

The morphology and state of nanoparticle dispersion within the laminates was examined by SEM, and typical micrographs of PPS/ $\mathrm{IF}^{-\mathrm{WS}_{2}}(2.0 \mathrm{wt} \%) / \mathrm{CF}$ are displayed in Fig. 1. The lower magnification image (Fig. 1a) shows a CF tow, with an average fiber diameter of
$7 \mu \mathrm{m}$, surrounded by the PPS polymer. The matrix was observed to uniformly penetrate into the tow, and no open ring holes were found around the CFs, indicative of a good fiber-resin interfacial adhesion. The high magnification micrograph in Fig. 1b corresponds to a matrix rich region between fiber tows, showing the IF-WS 2 nanoparticles (bright spots) randomly dispersed through the polymer. These appear either as individual nanoparticles or as small aggregates of a few particles, and exhibit quasi-spherical shape with an average diameter of $85 \mathrm{~nm}$, in agreement with the value for as-received IF-WS 2 reported previously [19]. Moreover, some of the nanoparticles appeared to be partially buried in the PPS matrix, showing an apparent diameter smaller than the real size. Similar images were obtained from the other laminates, although the interparticle distance was found to decrease with increasing IF$W_{2}$ concentration, thereby raising the chance of nanoparticle flocculation once melt-mixing is stopped. Overall, the SEM analysis demonstrates the effectiveness of the melt-blending process to homogenously disperse the nanosized IF-WS 2 within a thermoplastic matrix without the aid of any surfactant or compatibilizing agent.

\subsection{Thermal stability}

The thermal stability of PPS/IF-WS $/$ /CF laminates was investigated using TGA. Typical thermograms under a nitrogen atmosphere are shown in Fig. 2, and the characteristic degradation temperatures of the different laminates under both inert and oxidative conditions are given in Table 2 . It was observed that all samples exhibit a single decomposition stage in a nitrogen environment, similar to that found for pure PPS, indicating that the random scission of the polymeric chains is the predominant degradation process [20]. However, the residual weight of the reference PPS/CF $(\sim 76 \%)$ is more than double that of the neat polymer ( $36 \%)$ [21], due to the presence of the CFs, and this residue rises progressively with the addition of increasing IF-WS 2 loadings, since these nanoparticles are thermally stable in nitrogen up to $800^{\circ} \mathrm{C}$ [19]. The incorporation of the IF-WS ${ }_{2}$ induces a thermal stabilization of the PPS matrix, with an increase in the initial degradation temperature $\left(T_{i}\right)$ of up to $12{ }^{\circ} \mathrm{C}$ at $2.0 \mathrm{wt} \%$ loading in comparison to the reference laminate. A similar trend was found for the temperature of maximum rate of weight loss $\left(T_{\max }\right)$, where the increments were larger, about $9,13,19$ and $24{ }^{\circ} \mathrm{C}$ for contents of $0.1,0.5,1.0$ and $2.0 \mathrm{wt} \% \mathrm{IF}_{-} \mathrm{WS}_{2}$, respectively. This behavior can be explained in terms of the barrier effect of the nanoparticles that effectively hinder the transport of volatile decomposed products from the bulk of the polymer to the gas phase, hence decelerating the decomposition process. With increasing IF-WS ${ }_{2}$ loading the barrier effect becomes stronger, which is reflected in higher degradation temperatures. An analogous behavior of thermal stability enhancement has been reported for
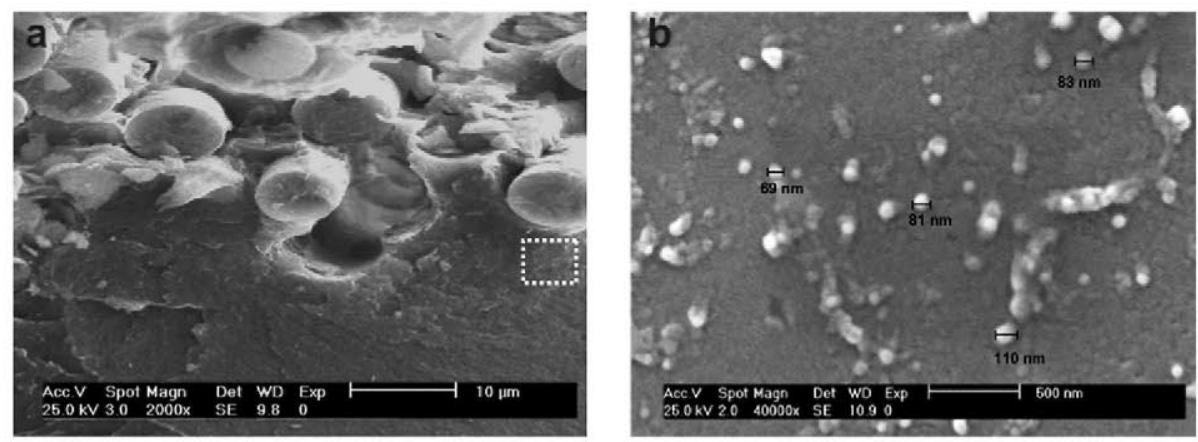

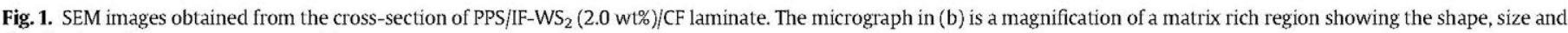
distribution of the IF-WS 2 nanoparticles. 


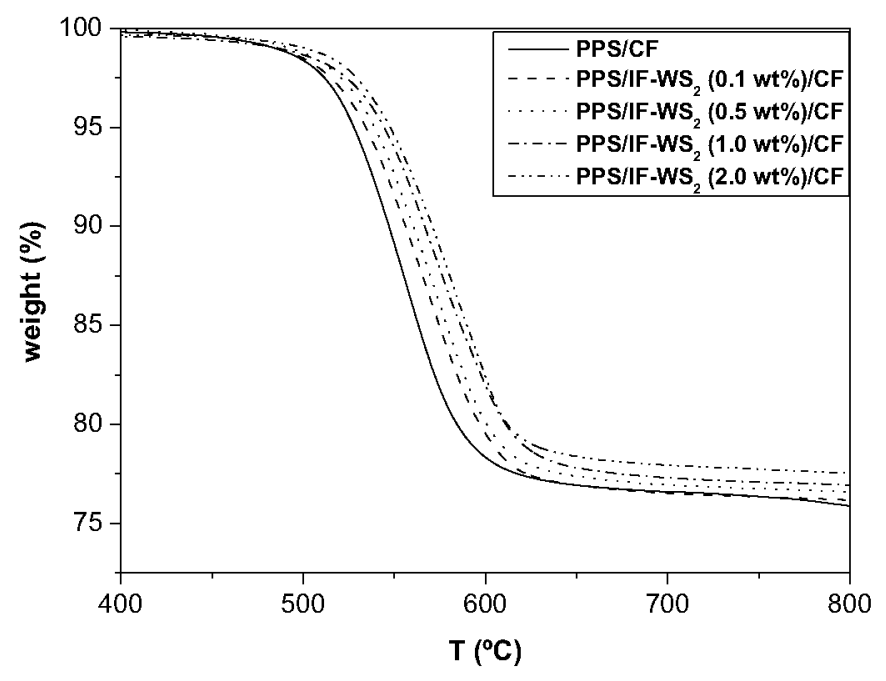

Fig. 2. TGA curves for PPS/CF and PPS/IF-WS $/$ /CF laminates under a nitrogen atmosphere at a heating rate of $10{ }^{\circ} \mathrm{C} / \mathrm{min}$, in the temperature range between 400 and $800^{\circ} \mathrm{C}$.

other polymer nanocomposites reinforced with spherical inorganic nanoparticles such as silica [22].

Regarding the degradation under an oxidative environment, two weight loss stages were detected that finally lead to the total decomposition of the material before $800{ }^{\circ} \mathrm{C}$; the first is attributed to the scission of the polymeric chains and the second to the decomposition of the CFs and the aromatic structures that remained in the residue under inert atmosphere. The thermal stabilization effect of the nanoparticles is qualitatively similar to that found under an inert atmosphere, with slightly smaller increments (Table 2). Thus, the addition of $2.0 \mathrm{wt} \%$ IF-WS 2 loading led to increases in $T_{i}$ and $T_{\max , I}$ of 8 and $13^{\circ} \mathrm{C}$, respectively. The smaller thermal enhancement under air compared to nitrogen should be related to the lower stability of the IF-WS 2 in that atmosphere, where they start to degrade at around $420{ }^{\circ} \mathrm{C}$ [19]. Nevertheless, the increments observed in this study are higher than those reported for binary PPS/IF-WS 2 nanocomposites [16], probably due to a synergistic stabilization effect arising from the presence of both fillers that have higher degradation temperatures than the neat matrix. The results clearly demonstrate that these laminates are suitable for high-temperature applications.

\subsection{Crystallization and melting behavior}

It is known [9] that crystallinity is of great importance in highperformance polymers because it has strong influence on both the chemical and mechanical properties. The crystalline phase improves the stiffness and tensile strength whilst the amorphous phase helps to absorb the impact energy. Therefore, it is important to analyze the influence of these nanoparticles on the crystallization and melting behavior of PPS/CF. The DSC cooling and melting curves of the different laminates are shown in Fig. 3, and the thermal parameters such as crystallization temperature $\left(T_{c}\right)$, melting temperature $\left(T_{m}\right)$ as well as the degrees of crystallinity obtained from the cooling and heating thermograms ( $\chi_{c}$ and $\chi_{m}$, respectively) are summarized in Table 3 . The value of $T_{C}$ for the reference laminate is $237^{\circ} \mathrm{C}$, about $6{ }^{\circ} \mathrm{C}$ lower than that of neat PPS [21]; a slight decrease is also observed in $T_{m}$ together with a reduction in $\chi_{c}$ and $\chi_{m}$. All these facts indicate that the CFs obstruct the diffusion and ordering of the PPS chains, decelerating the overall crystallization process.

Regarding the hybrid laminates, noticeable differences are found depending on the IF-WS ${ }_{2}$ concentration. In the case of low nanoparticle contents (i.e. 0.1 or $0.5 \mathrm{wt} \%$ ), the nucleation process of PPS crystals appears to be disrupted, leading to a decrease in $T_{c}, \chi_{c}$ and $\chi_{m}$ in comparison to those of the reference laminate, together with a slight shift in $T_{m}$ towards lower temperatures. This implies the absence of a nucleating effect of the IF-WS 2 on the polymer crystallization, and that the transport of macromolecular segments to the growing surface of PPS in the composite is difficult. However; further increase in the nanoparticle content provokes a rise in all the thermal parameters; for $2.0 \mathrm{wt} \%$ loading the increments in $T_{c}$, $T_{m}, \chi_{c}$ and $\chi_{m}$ are about 9 and $3{ }^{\circ} \mathrm{C}, 22$ and $14 \%$, respectively. This indicates that higher nanoparticle contents are effectively acting as nucleating agents for PPS, facilitating the crystallization of the molecular chains and increasing the crystallization rate. A similar change from inhibition to promotion in the crystallization of PPS with increasing nanoparticle content has been previously reported for PPS/IF-WS 2 binary nanocomposites [23]. However, in those composites the addition of $0.5 \mathrm{wt} \% \mathrm{IF}-\mathrm{WS} \mathrm{S}_{2}$ increased $T_{c}$, whereas in the case of these hybrid laminates, nanoparticle loadings equal or greater than $1.0 \mathrm{wt} \%$ are required to promote polymer crystallization, probably due to the presence of the CFs that inhibit the nucleating effect of the IF-WS $\mathrm{S}_{2}$. It can be noticed that the degrees of crystallinity obtained from the cooling curves are lower than those attained from the heating thermograms, which suggests the existence of reorganization phenomena and improvement (i.e. perfection and thickening) of the PPS crystals during the heating cycle subsequent to the crystallization process. Overall, the DSC data indicate that the desired degree of crystallinity can be achieved by controlling the IF-WS 2 concentration in the laminates.

Another feature that can be observed from the thermograms is the appearance of a small change in the heat capacity in the range of $90-130^{\circ} \mathrm{C}$ related to the glass transition process. This transition temperature $\left(T_{g}\right)$, which is about $100^{\circ} \mathrm{C}$ for the reference laminate, appears to decrease slightly for the hybrids incorporating low nanoparticle content, whereas it increases for those with IF-WS loadings $\geq 1.0 \mathrm{wt} \%$. The aforementioned change from reduction to acceleration in the crystallization rate of the matrix as the nanoparticle content increases should also be responsible for the different behavior observed in the glass transition. The $T_{g}$ values

Table 2

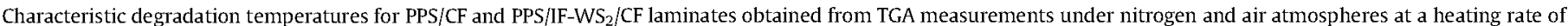
$10^{\circ} \mathrm{C} / \mathrm{min}$.

\begin{tabular}{|c|c|c|c|c|c|c|c|}
\hline \multirow[b]{2}{*}{ IF-WS ${ }_{2}$ content $(w t \%)$} & \multicolumn{4}{|c|}{ Nitrogen } & \multicolumn{3}{|l|}{ Air } \\
\hline & $T_{\mathrm{f}}\left({ }^{\circ} \mathrm{C}\right)$ & $T_{10}\left({ }^{\circ} \mathrm{C}\right)$ & $T_{\max }\left({ }^{\circ} \mathrm{C}\right)$ & Residue $_{800}{ }^{\circ} \mathrm{C}(\mathrm{wt} \%)$ & $T_{i}\left({ }^{\circ} \mathrm{C}\right)$ & $T_{10}\left({ }^{\circ} \mathrm{C}\right)$ & $T_{\max 1, \mathrm{II}}\left({ }^{\circ} \mathrm{C}\right)$ \\
\hline 0 & 502 & 546 & 555 & 75.9 & 460 & 509 & 527,606 \\
\hline 0.1 & 503 & 553 & 564 & 76.1 & 460 & 510 & 526,609 \\
\hline 0.5 & 507 & 558 & 568 & 76.6 & 463 & 515 & 532,613 \\
\hline 1.0 & 508 & 564 & 574 & 77.0 & 465 & 519 & 536,615 \\
\hline 2.0 & 514 & 567 & 579 & 77.7 & 468 & 523 & 540,617 \\
\hline
\end{tabular}

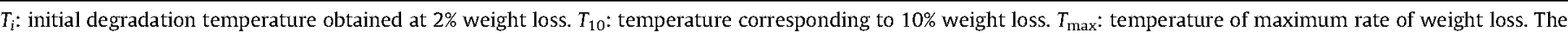
subscripts I and II refer to the first and second degradation stages under air atmosphere, respectively. 


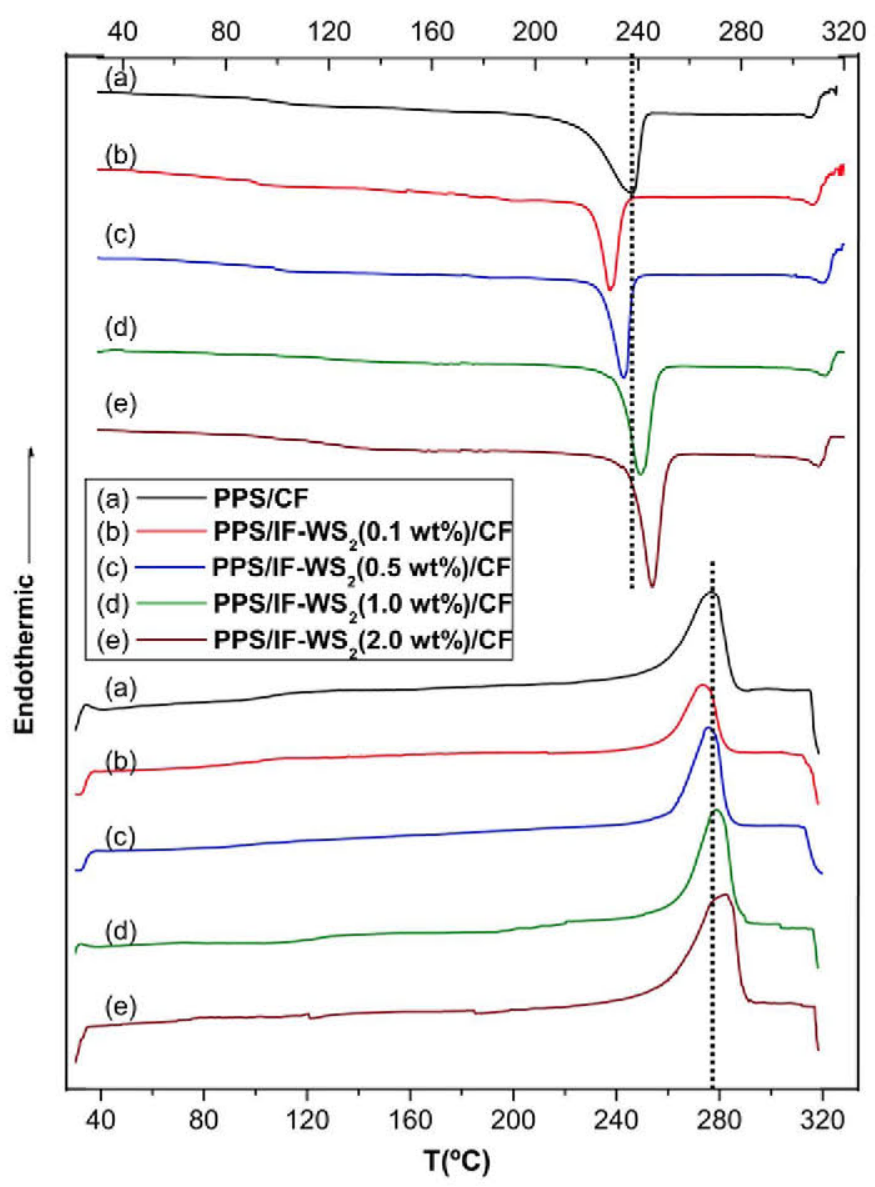

Fig. 3. Non-isothermal DSC cooling and heating thermograms for the different laminates obtained at a rate of $10^{\circ} \mathrm{C} / \mathrm{min}$.

have been more accurately determined from DMA measurements and will be discussed in the following section.

\subsection{Dynamic mechanical performance}

The influence of the IF-WS $S_{2}$ nanoparticles on the dynamic mechanical properties of PPS/CF was explored by DMA that provides information about the viscoelastic behavior of the matrix, indicating changes in the stiffness and the relaxation processes that occur as a function of temperature. Fig. 4 shows the effect of the nanoparticle content on the storage modulus $\left(E^{\prime}\right)$ and loss tangent $(\tan \delta)$ at the frequency of $1 \mathrm{~Hz}$. The value of $\mathrm{E}^{\prime}$ for the reference $\mathrm{PPS} / \mathrm{CF}$ at $25^{\circ} \mathrm{C}$ is $\sim 12.4 \mathrm{GPa}$ that corresponds to a 5.2 fold increase compared to that of neat PPS [16]. The addition of very low IF-WS 2 loadings (i.e. $0.1 \mathrm{wt} \%)$ leads to a slight decrease in $\mathrm{E}^{\prime}(\sim 7 \%)$ compared to the reference laminate. Taking into account that the storage modulus is highly affected by the composition and

Table 3

Parameters obtained from the DSC thermograms of PPS/CF and PPS/IF-WS $/$ /CF laminates.

\begin{tabular}{|c|c|c|c|c|}
\hline IF-WS 2 content (wt $\%$ ) & $T_{c}\left({ }^{\circ} \mathrm{C}\right)$ & $T_{m}\left({ }^{\circ} \mathrm{C}\right)$ & $\chi_{c}(\%)$ & $\chi_{m}(\%)$ \\
\hline 0 & 237.3 & 277.4 & 28.2 & 39.4 \\
\hline 0.1 & 229.8 & 274.9 & 22.1 & 32.9 \\
\hline 0.5 & 234.6 & 276.2 & 25.6 & 37.2 \\
\hline 1.0 & 241.2 & 278.6 & 30.4 & 40.5 \\
\hline 2.0 & 246.0 & 280.5 & 34.5 & 45.1 \\
\hline
\end{tabular}

$\overline{T_{c} \text { and } T_{m} \text { : crystallization and melting temperatures, respectively. } \chi_{c} \text { and } \chi_{m} \text { : degrees }}$ of crystallinity obtained from the cooling and heating thermograms. morphology of the material, this fall in $\mathrm{E}^{\prime}$ should be related to the decrease in the crystallinity found for this sample, as revealed by DSC data, since the crystalline regions enhance the modulus of semicrystalline polymers [24]. The laminate incorporating $0.5 \mathrm{wt} \%$ IF-WS 2 exhibits similar $E^{\prime}$ to that of PPS/CF, being only $\sim 5 \%$ higher at $25{ }^{\circ} \mathrm{C}$; the reinforcement effect of the $\mathrm{IF}^{-W S_{2}}$ probably compensates for the slight decrease in crystallinity, and the modulus is approximately maintained over the entire temperature range. In contrast, the incorporation of nanoparticle contents $>0.5 \mathrm{wt} \%$ resulted in significant $\mathrm{E}^{\prime}$ increments of $\sim 14$ and $22 \%$ for 1.0 and $2.0 \mathrm{wt} \% \mathrm{IF}-\mathrm{WS}_{2}$, respectively at $25^{\circ} \mathrm{C}$. These modulus enhancements are associated with the increase in crystallinity caused by heterogeneous nucleation, combined with the reinforcement effect. As the temperature increased from the glassy to the rubbery state, $E^{\prime}$ of all the laminates fell drastically, and the relative differences in modulus became slightly less pronounced.

The evolution of $\tan \delta$ (ratio of the loss to storage modulus, a measure of the damping within the system) as a function of temperature (Fig. 4) exhibits an intense peak, named $\alpha$ relaxation that corresponds to the $T_{g}$. The magnitude of the $\tan \delta$ peak is indicative of the filler-matrix interactions. In an unfilled system, the polymer chain segments are free from restraints. The incorporation of fibers decreases the free volume and restricts the mobility of the PPS chains, which is reflected in higher $T_{g}$ values. Thus, $T_{g}$ increased by $\sim 10^{\circ} \mathrm{C}$ with the addition of the CFs compared to that of the neat polymer $\left(\sim 90^{\circ} \mathrm{C}\right)[16]$.

Regarding the hybrid laminates, a different behavior was observed depending on the nanoparticle concentration. Composites with IF-WS 2 contents $\leq 0.5$ wt\% exhibited a downshift in $T_{g}$ compared to the reference laminate, whilst those with higher concentrations showed an upshift, in agreement with the results obtained from the DSC analysis. As mentioned earlier, the addition of low nanoparticle loadings reduces the crystallization rate of PPS, leading to the formation of a more amorphous phase that results in a slight decrease in $T_{g}$, of $\sim 8$ and $3{ }^{\circ} \mathrm{C}$ for 0.1 and $0.5 \mathrm{wt} \% \mathrm{IF}-\mathrm{WS}_{2}$, respectively. In contrast, the incorporation of higher contents has a nucleation effect, thereby raising the crystallinity of PPS that, combined with a larger IF-WS ${ }_{2}$-matrix interfacial contact area, results in an effective immobilization of the polymer chains, hence

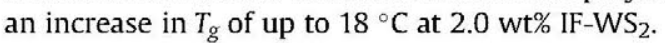

The damping in the transition region measures the amount of energy used to deform the material that is directly dissipated into heat. In comparison with neat PPS [16], the height of the $\tan \delta$ peak decreased in the presence of the CFs, since these hinder the movement of the polymer molecules. Moreover, the incorporation of IF$\mathrm{WS}_{2}$ contents $>0.5 \mathrm{wt} \%$ led to an additional reduction in $\tan \delta_{\max }$, indicating a decrease in the mechanical loss to overcome friction between molecular chains. The fall in the peak height was about $33 \%$ for the laminate with $2.0 \mathrm{wt} \%$ loading, pointing out the effectiveness of high IF-WS $\mathrm{W}_{2}$ contents to hamper molecular movement. Moreover, this remarkable decrease probably arises from a synergistic effect between both types of fillers on the restriction of chain mobility, in agreement with the behavior reported for other hybrid composites (i.e. epoxy/Ag/CNT) [25]. It can also be observed that the $\tan \delta$ peak broadens with increasing nanoparticle loading. This broadening can be interpreted as increased nanofiller-PPS interactions and is indicative of a larger nanoparticle-matrix interfacial area. The IF-WS 2 and CFs perturb the relaxation of the neighboring polymer chains that would behave differently from those situated in the bulk matrix, resulting in a wider maximum. The aforementioned effect has also been attributed to a more inhomogeneous amorphous phase in the composites in relation to the pure matrix [26]. Overall, the DMA results demonstrate that the IF-WS $\mathrm{WS}_{2}$ nanoparticles are suitable candidates to improve the dynamic mechanical properties of conventional thermoplastic/CF laminates. 


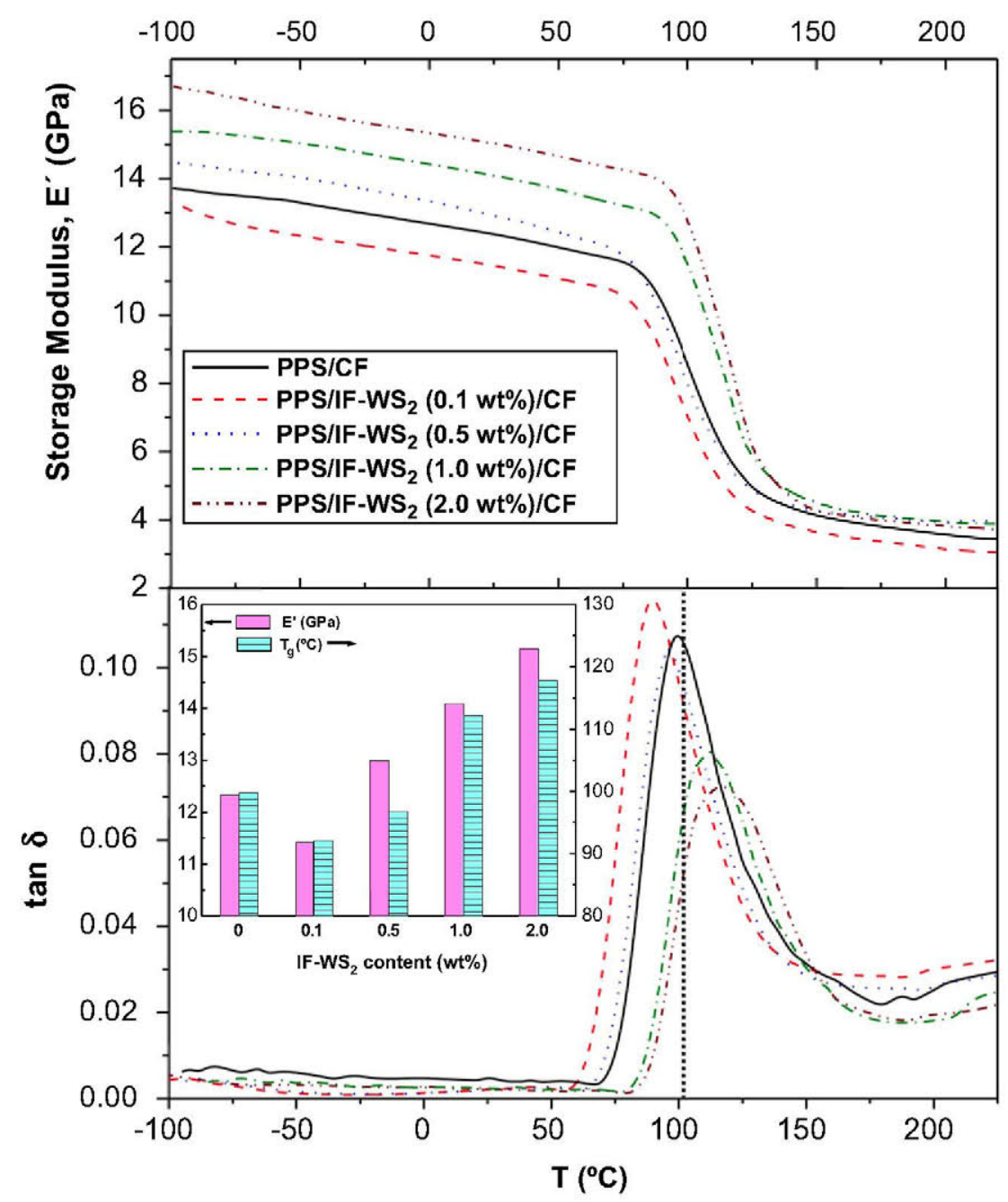

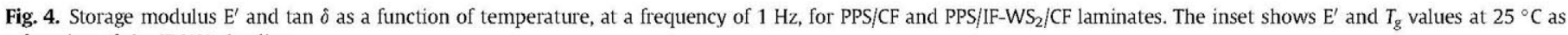
a function of the IF-WS $\mathrm{S}_{2}$ loading.

\subsection{Tensile and flexural tests}

The tensile properties of the laminates were also investigated, and the Young's modulus $(E)$, tensile strength $\left(\sigma_{y}\right)$, elongation at break $\left(\varepsilon_{b}\right)$ and toughness $(T)$ obtained from the room temperature stress-strain curves are plotted in Fig. 5 as a function of the nanoparticle loading. $E$ of neat PPS ( $\sim 2.2 \mathrm{GPa})$ [17] experiences about a 6.3 fold enhancement with the addition of the CFs (Fig. 5a), indicating that the stress is efficiently transferred from the matrix to the microscale fillers. Regarding the multiscale composites, the trend observed is qualitatively similar to that found from the DMA measurements, with a slight decrease $(\sim 5 \%)$ at $0.1 \mathrm{wt} \%$ nanoparticle content, and a progressive increment at higher loadings, up to $16 \%$ at $2.0 \mathrm{wt} \% \mathrm{IF}-\mathrm{WS}_{2}$. This points out that the acceleration of the crystallization rate of PPS due to the nucleating effect of the IF-WS 2 promotes the formation of small imperfect crystals that provide high rigidity to the semicrystalline PPS [27]. An analogous behavior of modulus improvement has been reported previously for CF-reinforced epoxy composites with dispersed nanosized $\mathrm{Al}_{2} \mathrm{O}_{3}$ particles [12].

On the other hand, the tensile strength (Fig. 5b) of the laminate incorporating $0.1 \mathrm{wt} \% \mathrm{IF}_{-} \mathrm{WS}_{2}$ is similar to that of the reference PPS/ $\mathrm{CF}$, and increases moderately with the nanoparticle content, the increase being around $12 \%$ for the highest concentration tested. The results indicate that these inorganic nanoparticles contribute more to the enhancement of the stiffness than the strength of the polymer. Focusing on the elongation at break (Fig. 5c), a strong dependence on the nanofiller content was observed. Composites reinforced with low IF-WS 2 loadings ( $\leq 0.5 \mathrm{wt} \%$ ) that possess lower degree of crystallinity than the reference laminate exhibited higher ductility. The spherical shape of the nanoparticles and their very homogenous distribution within the matrix (hence large interparticle distance) hardly hinder the ductile flow of the polymer chains. In contrast, when a greater amount of nanoparticles was added, the tensile elongation started to decrease due to the formation of small particle aggregates (revealed by SEM) that restrict the plastic deformation of the matrix and lead to more hindrance when contacting with the polymer segments. A qualitatively similar behavior was found for the toughness (Fig. 5d), measured as the area under the tensile curve. Nevertheless, the maximum improvement in $T$ $(\sim 23 \%)$ is attained at $0.5 \mathrm{wt} \%$, whereas for $\varepsilon_{b}$ the largest increase corresponds to the composite with $0.1 \mathrm{wt} \% \mathrm{IF}_{-} \mathrm{WS}_{2}$. Hybrids with nanofiller loading $>1.0 \mathrm{wt} \%$ exhibit lower toughness than PPS $/ \mathrm{CF}$, since the presence of small agglomerates results in higher stress concentration at the particle-matrix interface, thereby leading to premature failure.

The Young's modulus of polymer/nanofiller/fiber multiphase composites can be predicted by a two-phase model. Firstly, considering $E$ of the IF-WS (Table 1), the corresponding values of binary polymer/nanoparticle composites can be calculated according to the classical rule of mixtures. The PPS/IF-WS 2 mixture 

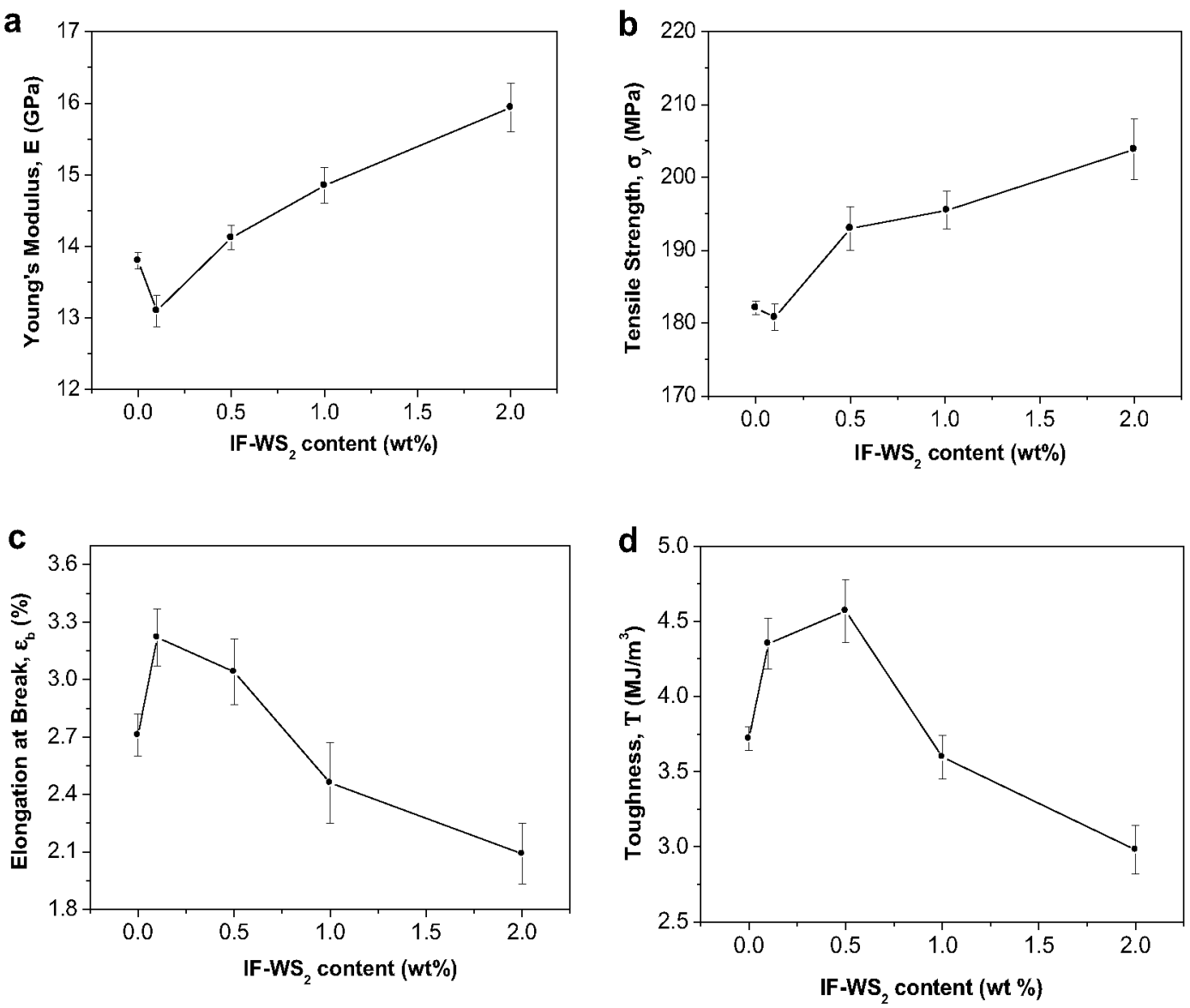

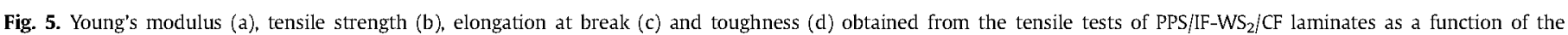
nanoparticle loading.

can then be regarded as a new matrix phase and the CFs as the filler phase; the rule of mixtures can be subsequently used to calculate the Young's modulus of the laminates: $E_{c}=V_{f} E_{f}+\left(1-V_{f}\right) E_{m}$, where $E_{f}$ and $E_{m}$ are the fiber and matrix modulus, respectively, and $V_{f}$ the fiber volume fraction. For all the laminates prepared the estimated moduli considerably exceed the experimental data; the discrepancies should arise from several factors such as the very high viscosity of the resin, which precludes complete fiber wetting; hence the fibers are not able to develop their full capability for stiffness enhancement. Moreover, it should be taken into account that the equation employed describes an upper limit expected in the case of perfect nanoparticle dispersion and adhesion to the polymer, perfect fiber-matrix bonding and zero inter-tow gap.

The results obtained from the room temperature flexural tests are displayed in Fig. 6. The flexural modulus $\left(E_{f}\right)$ of the hybrid incorporating $0.1 \mathrm{wt} \% \mathrm{IF}^{-\mathrm{WS}_{2}}$ is slightly lower than that of the reference laminate, while it increases progressively at higher

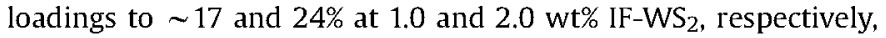
mirroring the Young's modulus behavior. Analogously, the flexural strength data $\left(\sigma_{f M}\right)$ follow similar trend to that described previously for the tensile strength, albeit with larger increments of $\sim 14$ and $17 \%$ for the aforementioned loadings. For multiscale composites it is expected that the nanoscale reinforcement primarily influences the properties that are matrix-dominated [10]. The remarkable improvement in $E_{f}$ obtained upon addition of the nanoparticles is attributed to the reinforcement effect of the IF-WS 2 in the $z$ direction, since the flexural properties are mainly matrixdominated. However, a moderate increase is achieved in the Young's modulus that is mostly fiber-dominated.

\subsection{Charpy impact strength}

Fig. 7 presents the results obtained from room temperature Charpy notched impact tests. Neat PPS is brittle and exhibits a low impact strength $\left(G \sim 1.7 \mathrm{~kJ} / \mathrm{m}^{2}\right)$ [28], which has limited many of its potential applications. The addition of the CFs increased its impact strength by about $40 \%$, in agreement with the behavior reported for different CFRP [29]. Further incorporation of small amounts of IF$W_{2}$ resulted in an additional rise in $G$, and the maximum

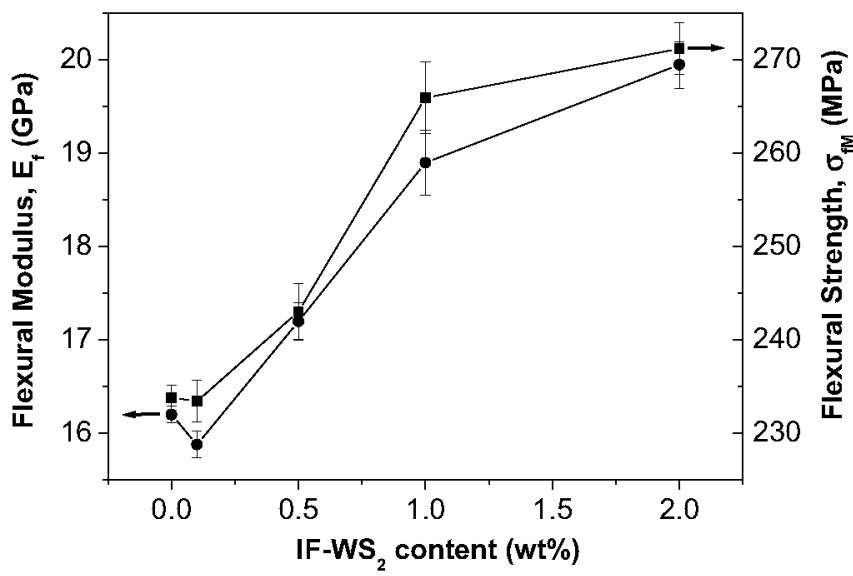

Fig. 6. Effect of the IF-WS $\mathrm{S}_{2}$ concentration on the room temperature flexural modulus and strength of PPS/IF-WS $/$ CF laminates. 
improvement of 24\% compared to PPS/CF was attained at $0.1 \mathrm{wt} \%$ loading. The hybrid reinforced with $1.0 \mathrm{wt} \% \mathrm{IF}^{-\mathrm{WS}_{2}}$ showed similar impact strength to that of the reference laminate, whereas higher loading led to a decrease of around $12 \%$. These results are consistent with the toughness data obtained from the tensile tests; for each nanoparticle concentration tested the variation of energy absorbed during the impact test in relation to the reference PPS/CF differs by less than $10 \%$ from the corresponding change in toughness calculated from the areas under the stress-strain curves. It has been reported [28] that there is an inverse relationship between the impact resistance and the degree of crystallinity of PPS. The addition of nanoparticle loadings $\leq 0.5 \mathrm{wt} \%$ lowers the crystallinity of the matrix, as revealed by DSC analysis (Table 3 ), leading to an improvement in G. However, at higher contents the nanoparticles act as nucleating agents, thus the impact strength decreases.

Fig. 7 also shows the variation of the area under the $\tan \delta$ peak obtained from DMA measurements as a function of the IF-WS loading; composites with nanoparticle content $>0.5 \mathrm{wt} \%$ display smaller area than that for PPS/CF. Previous studies [30,31] correlated the impact strength and the dynamic mechanical properties in terms of the area under the $\tan \delta$ curve as this represents the energy dissipated in viscoelastic relaxations. The dependence of the impact strength of these hybrid composites on the IF-WS 2 concentration exhibits a qualitatively similar trend to that found for the area under the $\tan \delta$ peak. The results provided by both techniques indicate that the addition of very low nanoparticle contents increases the energy dissipation in the composite, improving its impact resistance, and the maximum enhancement is attained at $0.1 \mathrm{wt} \%$ loading.

\subsection{Microhardness and tribological properties}

The results of the Vickers microhardness (HV) measurements for the different laminates are shown in Fig. 8a. It can be observed that HV rises with increasing nanoparticle content, attributed to a hardening of the matrix by the addition of this nanoreinforcement, which is known to have a relatively high hardness of $\sim 0.6 \mathrm{GPa}$ [32]. It appears that the uniform dispersion of the spherical nanoparticles and the decrease in the interparticle distance with increasing IF-WS ${ }_{2}$ loading result in an improvement in the resistance to local plastic deformation, hence a rise in microhardness, the increment being $\sim 11 \%$ at $2.0 \mathrm{wt} \%{\mathrm{IF}-\mathrm{WS}_{2}}$ content. Nevertheless, at concentrations below $1.0 \mathrm{wt} \%$ the increase in microhardness is less significant and is almost negligible at

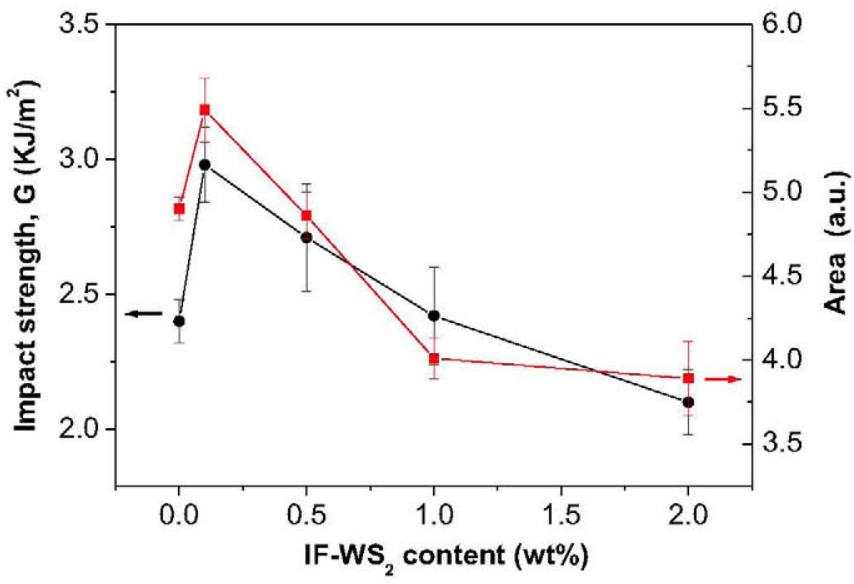

Fig. 7. Evolution of Charpy notched impact strength at $23^{\circ} \mathrm{C}$ and area under the tan $\delta$ peak obtained from DMA measurements as a function of the nanoparticle content for PPS/IF-WS $/$ /CF laminates.
$0.1 \mathrm{wt} \%$, probably due to the decrease in the crystallinity of the matrix. Low nanoparticle contents offer less resistance against the molecular movement of the PPS segments under indentation.

The addition of inorganic nanoparticles to thermoplastic polymers generally leads to an improvement in the tribological properties [33,34]. Fig. 8b displays the evolution of the coefficient of friction $(\mu)$ as a function of the IF-WS 2 content. The value of $\mu$ for neat PPS is around 0.46 [35], and decreases considerably with the addition of the CFs, attributed to the improvement in mechanical properties (i.e. modulus, strength, hardness, etc.) and the increase in thermal conductivity that lowers the temperature in the sliding contact, both factors reducing the indentation of the counterpart into the matrix. The incorporation of $0.1 \mathrm{wt} \% \mathrm{IF}^{-W S_{2}}$ leads to a slight increase in $\mu$ of $\sim 5 \%$ compared to the reference laminate,
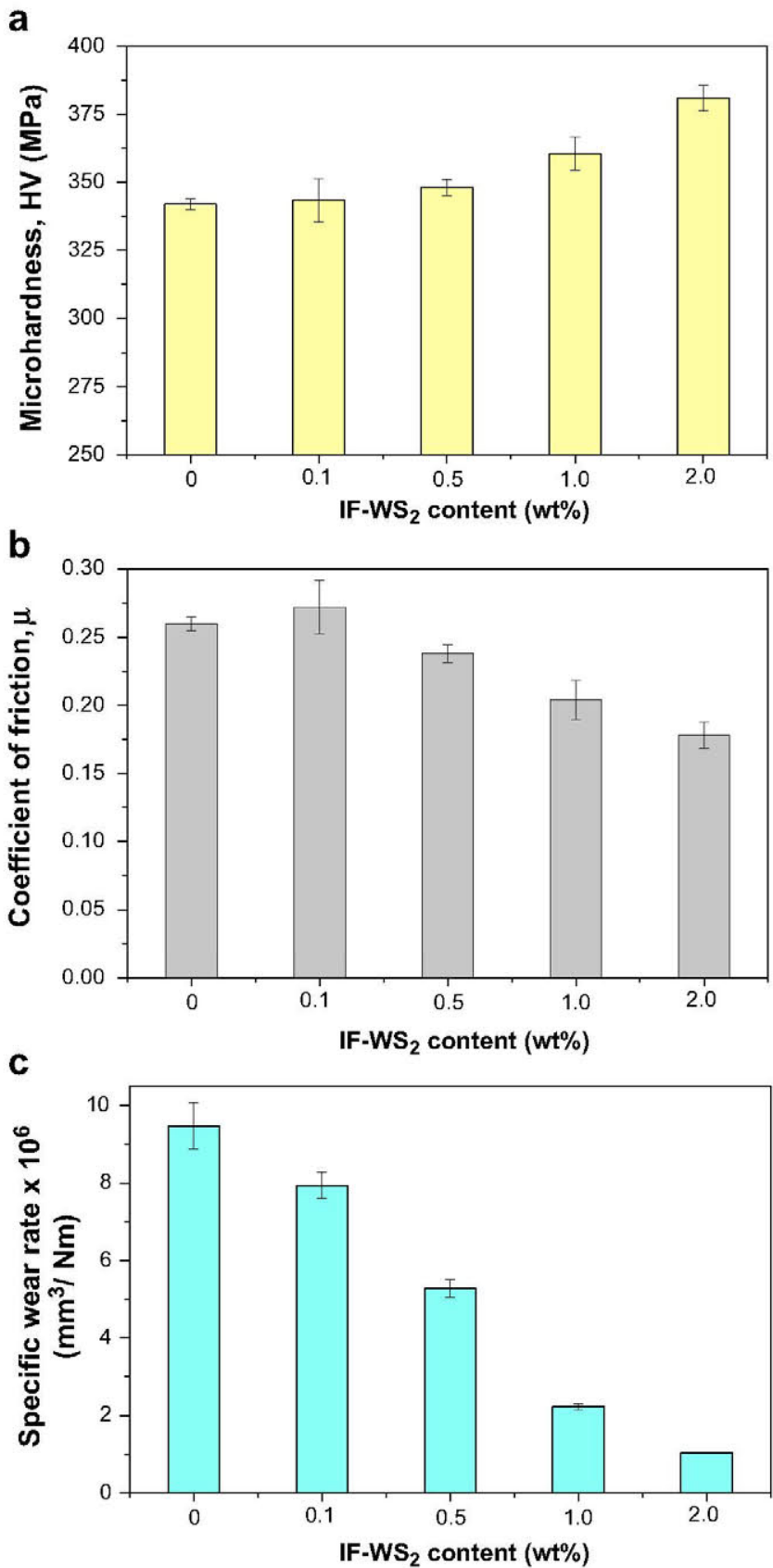

Fig. 8. Vickers microhardness (a), coefficient of friction (b) and specific wear rate (c) of the laminates prepared as a function of the nanoparticle content. 

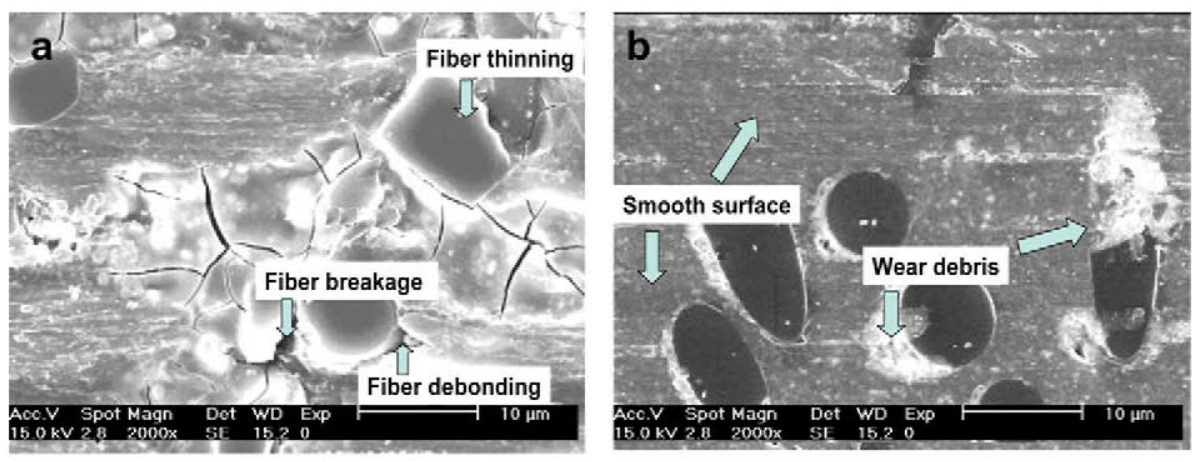

Fig. 9. Typical morphology of the worn surfaces of PPS/CF (a) and PPS/IF-WS 2 (1.0 wt\%)/CF (b) sliding against the steel counterpart.

probably ascribed to the decrease in stiffness and strength (revealed by tensile and flexural tests) that outweighs the lubricant effect of the IF-WS 2 . With further increase in the nanoparticle content, the coefficient of friction drops remarkably and reaches its lowest value of around 0.18 , a $32 \%$ drop, at $2.0 \mathrm{wt} \%$ loading, due to the enhanced mechanical performance combined with the lubricant effect. Rapoport et al. [36] have proposed a rolling mechanism for these nanoparticles; the IF-WS $\mathrm{W}_{2}$ act as a type of ball-bearing component, meaning that they roll rather than slide between the surfaces, effectively reducing the shear stress, coefficient of friction and contact temperature. A similar decreasing trend in $\mu$ due the addition of inorganic particles has been reported for different hybrid systems such as $\mathrm{PEI} / \mathrm{CaSiO}_{3} / \mathrm{CF}$ composites [37], which was ascribed to the decrease in the shear forces and the enhanced loadbearing capacity. Moreover, the aforementioned behavior can also be attributed to synergistic effects between the fibers and the nanoscale fillers, as previously described for CF-reinforced PEEK incorporating $\mathrm{ZnS}$ or $\mathrm{TiO}_{2}$ nanoparticles [38].

The wear rate of PPS/CF (Fig. 8c) is much lower (about a factor of $10^{2}$ ) than that of the neat matrix, in agreement with the behavior reported by Cho et al. [35] for CNF-reinforced PPS composites. Regarding the different hybrids, a progressive reduction in this parameter was found with increasing IF-WS $\mathrm{S}_{2}$ concentration, decreasing by approximately a factor of 9 for the composite with $2.0 \mathrm{wt} \%$ loading compared to the reference laminate. This increase in wear resistance is ascribed to the formation of a continuous, smooth and thin transfer film on the counterface during sliding combined with the reinforcing effect, and it is enhanced by the presence of the two fillers. The adhesion of the transfer film is expected to improve as a uniform mixture of the debris is formed, and the resistance to microcracking as well as fatigue failure may also be enhanced by the nanoparticles. A similar trend was reported for the wear behavior of $\mathrm{PEEK} / \mathrm{ZrO}_{2} / \mathrm{CF}$ composites [11], where a synergistic effect of CFs with $\mathrm{ZrO}_{2}$ nanoparticles on the enhancement of the sliding wear resistance was proposed. Based on the results obtained, the combination of conventional CFRP with nanoscale lubricant fillers such as IF-WS appears to be a promising route to further optimize the tribological performance of the resulting hybrid composites.

To obtain more information about the wear mechanism of the composites and the influence of the IF-WS 2 on the wear rate, the worn surfaces of PPS/CF and PPS/IF-WS $2(1.0 \mathrm{wt} \%) / \mathrm{CF}$ were observed by SEM, and typical micrographs are displayed in Fig. 9. It is well known that the wear performance of CF-reinforced composites is governed mainly by fiber thinning, debonding and breakage under dry-sliding conditions [11]. After wear of the matrix material on and around the fibers, these are exposed to the counterpart asperities and are thinned down, subsequently leading to cracks and fiber debonding from the matrix. These phenomena can be visualized in the micrograph of PPS/CF (Fig. 9a). Nevertheless, in the composite with both types of fillers (Fig. 9b), the worn surface is found to be considerably smoother, with less pronounced scratches in comparison to that of the reference composite. Moreover, a greater amount of wear debris is accumulated around the CFs that separates the composite surface from the counterpart, thus protecting the fibers. Consequently, fiber breakage and debonding are minimized by the presence of the nanoparticles, which are key factors for enhancing the wear resistance.

\section{Conclusions}

The effect of IF-WS $S_{2}$ nanoparticles on the thermal, mechanical and tribological properties of PPS/CF laminates has been studied. SEM revealed a uniform dispersion of the nanoparticles within the matrix without the aid of surfactants or compatibilizing agents. TGA studies demonstrated a significant thermal stability enhancement in the presence of the IF-WS 2 . A strong dependence of the crystallization temperature and degree of crystallinity on the nanoparticle concentration was observed; $\mathrm{IF}_{-} \mathrm{WS}_{2}$ contents lower than $1.0 \mathrm{wt} \%$ decreased the crystallization rate of the matrix, whilst higher loadings effectively acted as nucleating agents for PPS in the $\mathrm{CF}$ composites. This change from inhibition to promotion was also reflected in the mechanical properties; the addition of very small amounts of IF-WS 2 decreased slightly the storage, tensile and flexural moduli as well as the glass transition temperature of PPS/ $\mathrm{CF}$, whereas they increased remarkably at higher concentrations, particularly the out-of-plane mechanical properties (i.e. up to $24 \%$ improvement in the flexural modulus at 2.0 wt\% loading). An optimum balance between stiffness and toughness was attained at $1.0 \mathrm{wt} \% \mathrm{IF}-\mathrm{WS}_{2}$. The microhardness of the composites increased steadily with the nanoparticle content, whilst a strong reduction in the coefficient of friction and wear rate was observed. The excellent wear resistance of the hybrid composites was believed to be due to a synergistic effect between the IF-WS 2 and the CFs. Overall, the results obtained confirm that although the IF-WS $\mathrm{W}_{2}$ nanoparticles constitute a very small weight fraction of the laminates as a whole, they are able to modify their macroscopic mechanical and tribological properties, leading to simultaneous improvements in stiffness, strength, hardness and wear resistance compared to the reference PPS/CF. The incorporation of both micro- and nanoscale fillers in a thermoplastic matrix opens up new perspectives for the development of multifunctional materials to be used in tribological components or high-performance structures such as wind turbine blades, aircraft and automobile elements, pressure vessels, to mention but a few.

\section{Acknowledgment}

Financial support from the Ministerio de Ciencia e Innovación (MICINN, Project MAT2010-21070-C02-01) is gratefully acknowledged. MN would like to acknowledge the Consejo Superior de 
Investigaciones Cientificas (CSIC) for a Postdoctoral Contract through Intramural Project No. 201160E003 and the Ministerio de Economía y Competitividad (MINECO) for a Ramon y Cajal research fellowship.

\section{References}

[1] Offringa AR. Thermoplastic composites in aerospace-proven through costeffective processing. In. 41th international conference on automated composites (ICAC). Nottingham, UK; 1995. pp. 161-169.

[2] Marsh G. Mater Today 2003;6:36-43.

[3] Brondsted P, Lilholt H, Lystrup A. Annu Rev Mater Res 2005;35:505-38.

[4] Troughton M]. Handbook of plastics joining: a practical guide. 2nd ed. New Yersey: William Andrew Inc; 2008.

[5] Brady DG. J Appl Polym Sci Appl Polym Symp 1981;36:231-9.

[6] Wayne H, Hill HW, Brady DG. Encyclopaedia of polymer science and engineering, vol. 11. New York: Willey; 1988. p. 531-532

[7] Mitschang P, Blinzler M, Wöginger A. Compos Sci Tech 2003;63:2099-110.

[8] De Baere I, Van Paepegem W, Degrieck J. Polym Compos 2009;30:1016-26.

[9] Vieille B, Aucher J, Taleb L. Mater Sci Eng A 2009;517:51-60.

[10] Kim M, Park Y-B, Okoli Ol, Zhang C. Compos Sci Tech 2009;69:335-42.

[11] Lin G, Xie G, Sui G, Yang R. Compos Part B 2012;43:44-9.

[12] Hussain M, Nakahira A, Nishijima S, Niihara K. Compos Part A 2003;31:173-9.

[13] Timmerman JF, Hayes BS, Seferis JC. Compos Sci Tech 2002;62:1249-58.

[14] Tsantazalis S, Karapappas $P$, Vavouliotis A, Tsotra P, Kostopoulos V, Tanimoto T. Compos Part A 2007;38:1159-62.

[15] Tenne R, Margulis L, Genut M, Hodes G. Nature 1992;360:444-6.

[16] Naffakh M, Marco C, Gomez MA, Gomez-Herrero J, Jimenez I. J Phys Chem B 2009;113:10104-11.
[17] Díez-Pascual AM, Naffakh M, Marco C, Ellis G. Compos Part A 2012;43 603-12.

[18] Tevet $O$, Goldbart $O$, Cohen SR, Rosentsveig R, Popovitz-Biro R, Wagner HD et al. Nanotechnology 2010;21:365705-11.

[19] Naffakh M, Martin Z, Fanegas N, Marco C, Gomez MA. J Polym Sci Part B Polym Phys 2007;45:2309-21.

[20] Budgell DR, Day M, Cooney JD. Polym Deg Stab 1994;43:109-15.

[21] Diez-Pascual AM, Guan J, Simard B, Gomez-Fatou MA. Compos Part A 2012;43 997-1006.

[22] Wen X, Zhang K, Wang Y, Han L, Han C, Zhang H, et al. Polym Int 2011;60: 202-10.

23] Naffakh M, Marco C, Gomez MA, Jimenez I. J Phys Chem B 2009;113:7107-15.

[24] Seymour RB, Carraher CE. Structure - property relationship in polymers. London: Plenum Press; 1984.

[25] Marcq F, Demont P, Monfraix P, Peigney A, Ch Laurent, Falat T, et al. Microelectron Reliab 2011:51:1230-4.

[26] Díez-Pascual AM, Ashrafi B, Naffakh M, Gónzalez-Domínguez JM, Johnston A Simard B, et al. Carbon 2011;49:2817-33.

27] Lu SX, Cebe P. J Appl Polym Sci 1996;61:473-83.

[28] Lu D, Mai Y-W, Li RKY, Ye L. Macromol Mater Eng 2003;288:693-8.

[29] Chand S. J Mater Sci 2000;35:1303-13.

[30] Gupta AK, Purwar SN. J Appl Polym Sci 1986;31:535-51.

[31] Jafari SH, Gupta AK. J Appl Polym Sci 2000;78:962-71.

[32] Nossa A, Cavaleiro A. Surf Coating Tech 2003;163-164:552-60.

[33] Wang Q, Xue Q, Liu H, Shen W, Xu J. Wear 1996;198:216-9.

[34] Hou X, Shan CX, Choy K-L. Surf Coating Tech 2008;202:2287-91.

[35] Cho MH, Bahadur S. Tribol Lett 2007;25:237-45.

[36] Rapoport L, Fleischer N, Tenne R. Adv Mater 2003;15:651-5.

[37] Xian G], Zhang Z, Friedrich K. J Appl Polym Sci 2006;101:1678-86.

[38] Oster F, Haupert F, Friedrich K, Muller M, Bickle W. Materialwiss Werkst 2004; 35:690-5. 